Research Article

Open Access

\title{
A new method for aldo-sugar analysis in beverages and dietary foods
}

\section{Wei-Ting Hung ${ }^{1}$, Yi-Ting Chen ${ }^{1}$, Shwu-Huey Wang ${ }^{2}$, Yin-Chen Liu ${ }^{3}$ and Wen-Bin Yang ${ }^{1}$}

${ }^{1}$ Genomics Research Center, Academia Sinica, 128 Academia Road, Section 2, Taipei 115, Taiwan; ${ }^{2}$ Core Facility Center, Office of Research and Development, Taipei Medical University, $250 \mathrm{Wu}-H \operatorname{sing}$ Street, Taipei 110, Taiwan; ${ }^{3}$ Sugarlighter Corporation, 3F., No.16-1, Mingfeng Street, New Taipei City 221, Taiwan

Corresponding author: Wen-Bin Yang, PhD, Professor, Genomics Research Center, Academia Sinica, Taiwan

Submission Date: October 27, 2015, Acceptance Date: April 24, 2016, Publication Date: April 27, 2016

\begin{abstract}
Background: Carbohydrates are a common part of our everyday diet. However, sugar analysis is difficult and inconvenient when it comes to food materials such as beverages, fruits, and vegetables. Consequently, in our article we discuss a new method for labeling the sugar ingredients in beverages and plant foods. This method provides a highly sensitive and efficient tool for sugar compositional analysis by labeling the aldoses in beverages and foods with 2,3naphthalenediamine via an iodine-promoted oxidative condensation reaction to form highly fluorescent aldo-naphthylimidazole (NAIM) derivatives. We have also separated the natural glycosides from dietary foods: for example, solanines from tomato and potato. The various types of solanines with different sugar moieties were analyzed by matrix-assisted laser desorption/ionization time-of-flight mass spectrometry (MALDI-TOF MS) and electrospray ionization tandem mass spectrometry (ESI-MS/MS). The solanine glycans were released by acidic hydrolysis, and the sugar components were subjected to NAIM labeling. These aldo-NAIM derivatives not only demonstrated enhanced mass signaling, but also provided fluorescent moiety at the reducing end of sugar, thereby assisting detection in HPLC analysis.
\end{abstract}

Objective: To develop a rapid and sensitive sugar detection method for research and commercial use, as well as to determine the sugar composition in a selection of dietary beverages and functional foods.

Results: Five beverages in Taiwan were examined for the composition of six common sugars. 
Two Solanaceae samples extracted from the potato and tomato plants were measured by MALDI MS and ESI-MS/MS. The structures of solanines were elucidated and the glycan moieties were converted to the fluorescent NAIM derivatives to confirm their composition.

Conclusions: The results suggest that aldo-NAIM, accompanied with LC and MS, is an efficient and rapid method for evaluation of sugar composition and concentration in beverages and foods.

Key words: beverages, foods, potato, tomato, aldose, sugar analysis, fluorescence, NAIM kit

\section{INTRODUCTION:}

Carbohydrates are an essential part of the diet of living organisms. For example, sugar is normally consumed in households, being a common ingredient found in soft drinks, cookies, candies and other foods. However, for diabetics, sugar consumption must be regulated to avoid acute spikes in their blood sugar [1,2]. Moreover, various studies and research suggest that excessive intake of sugar for non-diabetics can induce chronic diseases and feed cancer cells [3, 4].

Based upon sugar intake recommendations and conclusions drawn from these studies, it is reasonable to conclude that dietary sweeteners are one of the causal factors in a chronic disease outcome [5]. These scientific recommendations by Dietary Guidelines Advisory Committee (DGAC) also align with and support the World Health Organization's (WHO) controversial guidelines on sugars [6]. Calories are the real issue of 'added sugars' in our dietary foods. In fact, the body of evidence from studies indicates that any observed effect of 'added sugars' on body weight is a function of total calories from all sources; there is no unique obesity property caused solely by 'added sugars' [7].

The fluorescent aldo-NAIM derivatives of saccharides can be analyzed by HPLC on a reversed phase column with improved detection sensitivity. The hydrophobic property of NAIM moiety also enhances the ionization efficiency in MS experiments [8, 9]. The Taiwan Food \& Drug Administration (TFDA) additionally proposes to regulate some common sugars (fructose, sucrose, glucose, galactose, lactose and maltose), as well as free sugar in foods. The beverages and food products must label the amounts of sugars as shown in declaration of the "Nutrition Facts Panel" (August 2015), similar to the standards currently recommended by FDA. This regulation will pose challenges for the food industry, even though it is unclear whether it will benefit consumers or not, according to a recent review published by the Institute of Food Technologists (IFT) [10]. Moreover, labeling 'added sugar' in beverages and foods is required, with the recommendation of advising consumers of the proper sugar intake to remain healthy resulting from and supporting these kinds of measures.

Solanaceae, in a nickname of nightshade, is an economically important family of flowering plants $[11,12]$. The family ranges from annual to perennial herbs, and includes a number of 
important agricultural crops, medicinal plants and spices. The Solanaceae family includes a number of commonly collected or cultivated species. The most economically important genus of the family is Solanum, which contains potato (S. tuberosum), tomato (S. lycopersicum) and eggplant (or aubergine, S. melongena). Some individuals from different cultures eat nightshade as a staple of their diet. Many members of the Solanaceae family contain potent alkaloids (e.g. solanines), which may also be highly toxic. The biological activities of solanines vary among different compositions of sugar. In our study, we used a new aldo-sugar labeling kit to enhance the LC and MS signals of sugar for food analysis.

\section{MATERIALS AND METHODS}

Food samples and reagents: Sugar samples and 2,5-dihydroxybenzoic acid (2,5-DHB) were purchased from Sigma-Aldrich Co., Llc. (Louisiana, MI). Beverages made by various brands of food companies were purchased from convenience stores. Fresh potatoes and tomatoes were obtained from the supermarkets in Taipei City, Taiwan. The NAIM kit used in this study was provided by Sugarlighter Co., Inc. (New Taipei City, Taiwan). Methyl alcohol, ethyl acetate and deuterium solvents were purchased from Merck \& Co., Inc. (Darmstadt, Germany). All chemicals were analytical grade and used without further purification. Double distilled water $\left(\mathrm{dd}-\mathrm{H}_{2} \mathrm{O}\right)$ and buffer solution were used throughout HPLC analysis.

Free glycan released from solanine: The plant material $(10 \mathrm{~g})$ was lyophilized and ground for homogenization. In a typical procedure for the release of free solanine from plant tissue, the dried plant material $(0.1 \mathrm{~g})$ was extracted using water/methanol $(3.0 \mathrm{~mL} ; 1: 2 \mathrm{v} / \mathrm{v})$ with vortexing in a glass tube and at room temperature for $2 \mathrm{~h}$. The resulting solution was dried in a SpeedVac, followed by an acid-catalyzed procedure used to release glycan from solanine. Afterwards, aqueous hydrochloride solution $(0.2 \mathrm{~mm}, 2.0 \mathrm{~mL})$ was added to the above extract, and the mixture was incubated for $16 \mathrm{~h}$ at room temperature. The resulting aqueous solution was lyophilized, and subjected to the fluorescent labeling experiment. The released glycan in the solanine hydrolysate was subsequently labeled with NAIM in order to instill the fluorescent derivative. The reaction was completed in $4 \mathrm{~h}$ at room temperature as indicated by the TLC analysis, with the mixture then being directly measured by ESI-MS.

Labeling glycan to fluorescent NAIM derivative: The lyophilized sugar mixture was directly labeled with NAIM kit following the published procedure $[8,9]$. The sugar mixture was briefly added to 2,3-naphthalenediamine $(1.0 \mathrm{mg})$ and iodine $(0.5 \mathrm{mg})$ in $\mathrm{AcOH}(1.0 \mathrm{~mL})$ at room temperature. The mixture was stirred for $4 \mathrm{~h}$ to complete the reaction, as indicated by the TLC analysis. The mixture was diluted by 20 times of mobile phase, and $10 \mu \mathrm{L}$ aliquot of sample solution was taken for HPLC analysis. Additionally, the standard curves of sugars (fructose, 
sucrose, glucose, galactose, lactose and maltose) in HPLC analysis for characterization of the concentrations of sugars in the samples was weighed in $1.0 \mathrm{mg}$ of monosaccharide or $2.0 \mathrm{mg}$ of disaccharide for NAIM labeling in acetic acid $(1.0 \mathrm{~mL})$ with NAIM labeling kit. After the reaction was complete ( $4 \mathrm{~h}$ ), the residue was diluted with 20 times of the mobile phase (ammonium formate, $50 \mathrm{~mm}, \mathrm{pH} 5.0$, containing $30 \%$ of $\mathrm{MeOH})$. The prepared solution $(10 \mu \mathrm{L}, 3.0 \mathrm{nmol})$ was directly analyzed by HPLC. No further purification was needed before analysis.

HPLC analysis: A LaChrom Elite HPLC (Hitachi, Japan) system equipped with ultraviolet (UV) and refractive index (RI) detectors were used for HPLC analysis. UV absorption at $310 \mathrm{~nm}$ or fluorescence at $360 \mathrm{~nm}$ emission (by $320 \mathrm{~nm}$ excitation) was used for detection. The aboveprepared NAIM-labeled aldoses were analyzed by chromatography on a C18 column $(4.6 \mathrm{~mm} \times$

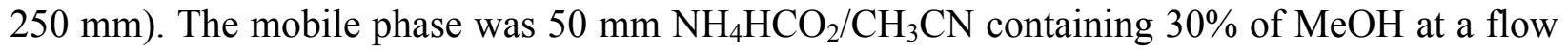
rate of $0.8 \mathrm{~mL} / \mathrm{min}$. The aldo-NAIM peaks were identified by comparison with the standards such as Glc-NAIM, Gal-NAIM, Lacto-NAIM and Malto-NAIM.

MALDI-TOF MS analysis: The MALDI-TOFMS spectra were acquired on an Ultraflex II MALDI-TOF/TOF mass spectrometer (Bruker Daltonik GmbH, Bremen, Germany). Typically, spectra were obtained by accumulating 800-1000 laser shots for quantitation. Laser power was fixed at $35 \%$ and the pulsed ion extraction was adjusted at $250 \mathrm{~ns}$. The mass range $(\mathrm{m} / \mathrm{z})$ was 600 $2100 \mathrm{Da}$, with the ion charge $\mathrm{z}=1.2,5$-DHB being used as the matrix.

LC-MS analysis: The NanoLC-ESI-FTMS experiments were used to monitor solanines and glycan-NAIMs. The experiments were performed on a LTQ Orbitrap XL ETD mass spectrometer (Thermo Fisher Scientific, San Jose, CA) equipped with a nanoelectrospray ion source (New Objective, Inc.); the Accela LC system was also used (Thermo Fisher Scientific, San Jose, CA). The sample solution ( $5 \mu \mathrm{L}$ ) was injected at $10 \mu \mathrm{L} / \mathrm{min}$ flow rate onto a self-packed pre-column $(150 \mu \mathrm{m}$ I.D. $\times 30 \mathrm{~mm}, 5 \mu \mathrm{m}, 200 \AA)$. Chromatographic separation was performed on a self-packed reversed phase $\mathrm{C} 18$ nano-column $(75 \mu \mathrm{m}$ I.D. $\times 200 \mathrm{~mm}, 2.5 \mu \mathrm{m}, 90 \AA)$, using $0.1 \%$ formic acid in water as mobile phase $\mathrm{A}$ and $0.1 \%$ formic acid in $80 \%$ acetonitrile as mobile phase $\mathrm{B}$ operated at $300 \mathrm{~nL} / \mathrm{min}$ flow rate with gradients from $10 \%$ to $40 \%$ of mobile phase B. A full-scan MS condition was applied with mass range $m / z 320-4000$, resolution 60,000 at $\mathrm{m} / \mathrm{z} 400$. Electrospray voltage was maintained at $1.8 \mathrm{kV}$ and capillary temperature was set at $200{ }^{\circ} \mathrm{C}$. All nanoLC-ESIFTMS was converted to $[\mathrm{M}+\mathrm{H}]^{+}$by using Xtract (Thermo Fisher Scientific, San Jose, CA) and all MS spectra were combined to single spectrum.

NMR analysis: The NMR spectra were recorded on a Bruker AV600 MHz NMR spectrometer (Bruker BioSpin $\mathrm{GmbH}$, Rheinstetten, Germany) with a $5 \mathrm{~mm}$ Dual cryoprobe $\mathrm{DCI}{ }^{1} \mathrm{H} /{ }^{13} \mathrm{C}$. 
Deuterium oxide (D2O) was used as solvent during the NMR experiment. The quantification of sugars was based upon the integral areas.

\section{RESULTS}

Characterization of six common sugars in beverages: Initially, we set the standard curves of sugars (fructose, sucrose, glucose, galactose, lactose and maltose) in HPLC analysis for characterization of the concentrations of sugars in the samples. The NAIM kit used in our study only tagged aldose at the reducing end. Therefore, the NAIM derivatives of lactose, glucose, galactose and maltose are shown using a UV detector (Figure 1A). Lacto-NAIM, Glc-NAIM, GalNAIM and Malto-NAIM were eluted out sequentially with retention times of 18.5, 19.9, 20.4 and 22.0 min, respectively, on a C18 HPLC column. When the sample contains keto-sugar (fructose) and non-reducing sugar (sucrose) together with aldose-NAIMs, the peaks are shown by using isocratic mobile phase and RI detector (Figure 1B). Although RI could be used to monitor six common sugars, including fructose and sucrose, higher concentration $(>1.0 \mu \mathrm{mol})$ of analyte was required than using UV detector.
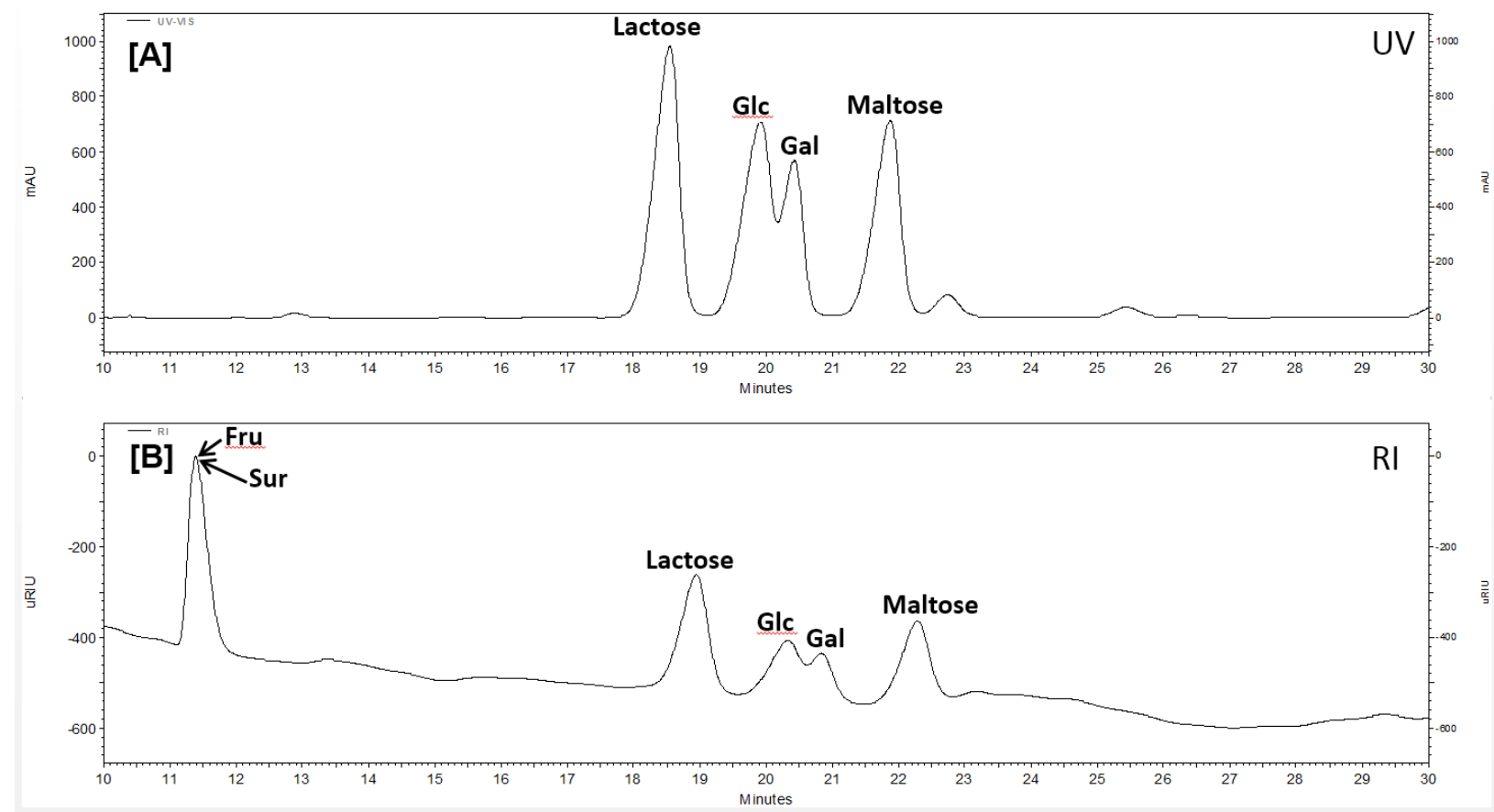

Figure 1. Characterization of six common sugars using UV (A) and RI (B) detectors in HPLC.

The glucose, galactose, lactose and maltose were derived to the corresponding aldo-NAIMs. Glc = glucose, Gal = galactose, $\mathrm{Fru}=$ fructose, Sur $=$ sucrose. After NAIM labeled the residue was diluted with 20 times of the mobile phase (ammonium formate, $50 \mathrm{~mm}, \mathrm{pH} 5.0$, containing 30\% of $\mathrm{MeOH}$ ). The prepared solution $(10 \mu \mathrm{L}, 3.0 \mathrm{nmol}) \mathrm{was}$ directly analyzed by HPLC. No further purification was needed before analysis.

The new aldo-NAIM method was applied to examine the composition of six common sugars 
in beverages (Table 1). Five types of beverages in Taiwan, including fruit juice, yogurt, coffee drink, milk tea and flavored milk, were examined.

Table 1. The ratio of sugars in common beverages by HPLC analysis of the sugar-NAIM derivatives using UV/RI detectors and NMR.

HPLC conditions: UV absorption at $310 \mathrm{~nm}$ or fluorescence at $360 \mathrm{~nm}$ emission (by $320 \mathrm{~nm}$ excitation) was used for detection. The prepared aldo-NAIMs were analyzed by chromatography on a C18 column $(4.6 \mathrm{~mm} \times 250 \mathrm{~mm})$. The mobile phase was $50 \mathrm{~mm} \mathrm{NH} \mathrm{HCO}_{2} / \mathrm{CH}_{3} \mathrm{CN}$ containing $30 \%$ of $\mathrm{MeOH}$ at a flow rate of $0.8 \mathrm{~mL} / \mathrm{min}$. The aldo-NAIM peaks were identified by comparison with the standards such as Glc-NAIM, Gal-NAIM, Lacto-NAIM and MaltoNAIM.

\begin{tabular}{|c|c|c|c|c|c|c|}
\hline $\begin{array}{c}\text { Sugar } \\
\text { Sample }\end{array}$ & Surcose & Fructose & Lactose & Glucose & Galactose & Maltose \\
\hline Orange A & 70 & 10 & - & 20 & - & $<1$ \\
\hline Grape B & - & 40 & - & 60 & - & - \\
\hline Fruit C & 35 & 18 & - & 47 & - & - \\
\hline Yogurt A & 3 & - & 1 & 56 & 39 & $<1$ \\
\hline Yogurt B & 49 & - & 1 & 26 & 24 & - \\
\hline Lactobacil. & 80 & - & 4 & 16 & - & - \\
\hline Coffee A & 89 & - & 9 & 2 & - & - \\
\hline Coffee B & 87 & - & 12 & 1 & - & - \\
\hline Coffee C & 80 & - & 15 & 5 & - & - \\
\hline Milk tea $\mathrm{A}$ & 90 & - & 6 & 4 & - & - \\
\hline Milk tea B & 68 & 5 & & 27 & - & $<1$ \\
\hline $\begin{array}{l}\text { Flavoured } \\
\text { milk A }\end{array}$ & 72 & - & 16 & 12 & - & - \\
\hline $\begin{array}{l}\text { Flavoured } \\
\text { milk B }\end{array}$ & 65 & - & 15 & 20 & - & - \\
\hline
\end{tabular}

In the fruit juice group (entries 1-3), the grape juice contained $40 \%$ fructose and $60 \%$ glucose, whereas orange juice and fruit C contained significant amounts of enriched sucrose, $70 \%$ and 35\% respectively. These results suggest that different fruits may contain different types of sugars in various compositions. In the lactic acid bacteria fermentation products (entries 4-6), yogurt A (original flavor) contains $56 \%$ of glucose and $39 \%$ of galactose without the addition of sucrose. Yogurt B (light sweet) has $49 \%$ of sucrose additive, whereas the lactobacillus fermented beverage appears to have a high content $(80 \%)$ of sucrose. Galactose was detected in yogurt A (entry 4 ), while yogurt B is enriched in glucose and galactose, which is generated by metabolism of lactose 
through bacteria fermentation (entry 5). It is notable that all of the tested samples of coffee (entries 7-9), milk tea (entries 10 and 11) and flavored milk (cocoa powder added, entry 12, and chocolate flavored, entry 13) appear to add high contents of sucrose to the original material and composition of the food products (e.g. black coffee or fresh milk). Except for milk tea B, other samples contain 6-16\% of lactose, which may be accounted for milk. In certain cases, we also used nuclear magnetic resonance (NMR) analysis to confirm the composition and ratio of six common sugars in beverages. The results from both HPLC and NMR analyses are consistent.

Characterization of the solanine sugars isolated from the potato and tomato plants: MALDI has been developed successfully as a soft ionization technique $[13,14]$. The benefit of MALDI mass spectrometry for analysis of saccharides resulted predominantly from the formation of singly charged molecular ions, which led to a simple mass spectral analysis for glycans and glycosides. In this study, the solanine samples extracted from the potato and tomato plants were measured by MALDI MS.

There are two major peaks at 853.0 and $869.0 \mathrm{Da}$ from the potato sample (Figure 2A) corresponding to solanines by comparison with literature data [15]. The chemical structure of the base peak at $m / z 869.0\left([\mathrm{M}+\mathrm{H}]^{+}\right)$was elucidated as the $\checkmark$-solanine $\left(\mathrm{C}_{45} \mathrm{H}_{73} \mathrm{NO}_{15}\right)$ bearing a trisaccharide substituent D-Glc(1,3)-D-Gal(1,2)-L-Rha on the alkaloid scaffold. A peak at $\mathrm{m} / \mathrm{z}$ 853.0 was attributable to the solanine of lower molecular weight $\left(\mathrm{C}_{45} \mathrm{H}_{73} \mathrm{NO}_{14}\right)$. For example, the weight suggested the sample contained a L-Rha-L-Rha-D-Gal substituent.

The MALDI MS spectrum of the tomato sample (Figure 2B) showed a major peak at $\mathrm{m} / \mathrm{z}$ 1072.8 that was assigned to the $[\mathrm{M}+\mathrm{H}]^{+}$ion of the solanine $\left(\mathrm{C}_{53} \mathrm{H}_{87} \mathrm{~N}_{2} \mathrm{O}_{20}\right.$; M.W. 1071.1) containing two hexoses, one $\mathrm{N}$-acetylhexosamine and one deoxyhexose. The solanine having the composition of three hexoses and one deoxyhexose occurred at $\mathrm{m} / \mathrm{z} 1034.8$. The structure for the MS signal at $m / z 1130.7$ remains to be elucidated.

By itself, MS is unable to identify the D-/L-configuration or the type of sugars, especially when distinguishing glucose from its stereoisomers of galactose and mannose. Furthermore, the linkages and anomeric configurations of the saccharides on the solanine scaffold cannot be identified just simply by using MS analysis. Alternatively, glycan-NAIM derivatives were prepared for NanoLC-ESI-FTMS experiments to monitor the glycan moiety of solanine (Figure $3)$. 


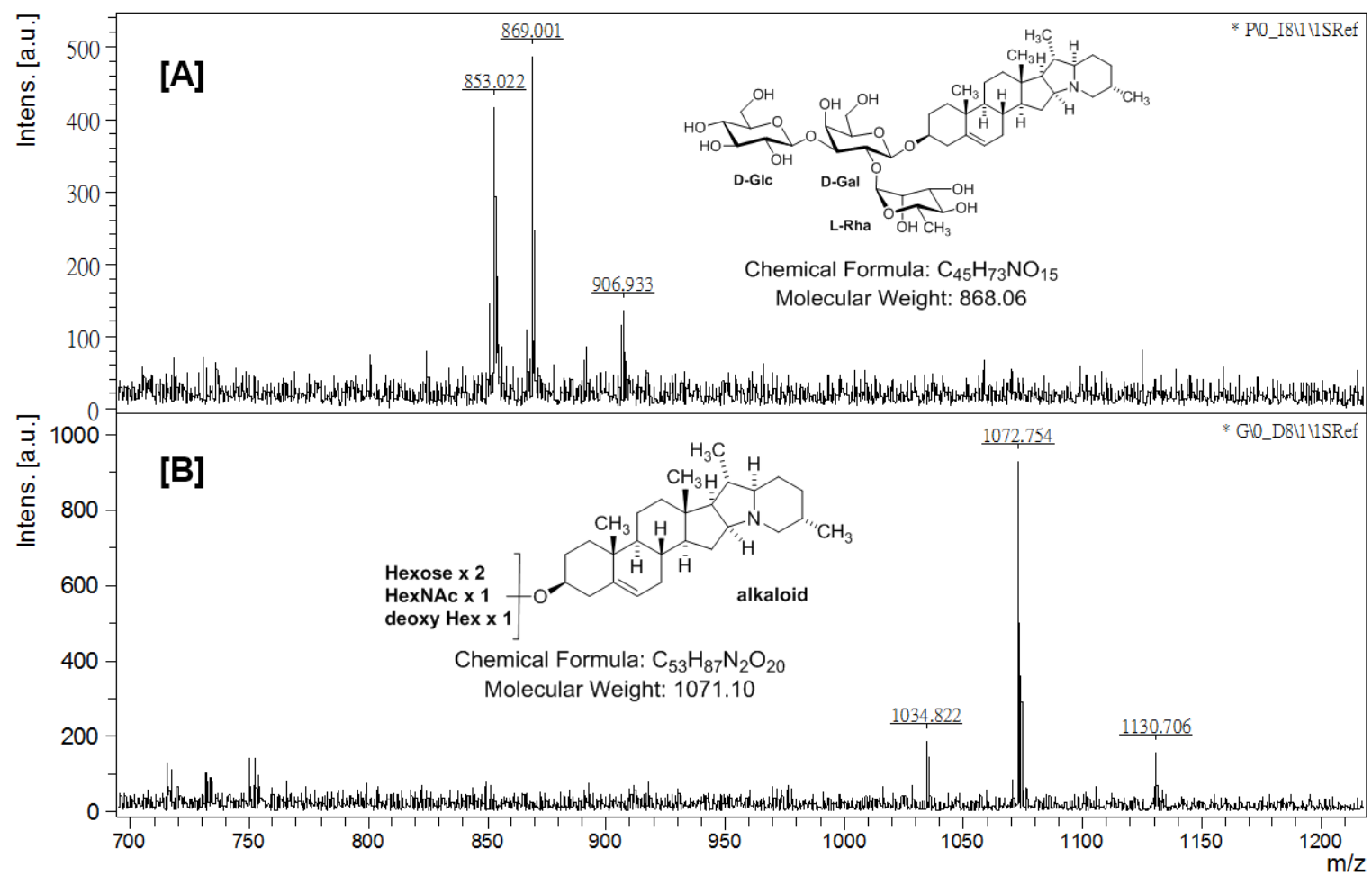

Figure 2. MALDI-TOF MS spectra of solanines. (A) fresh potato (Solanum tuberosum) and (B) tomato (Solanum lycopersicum).

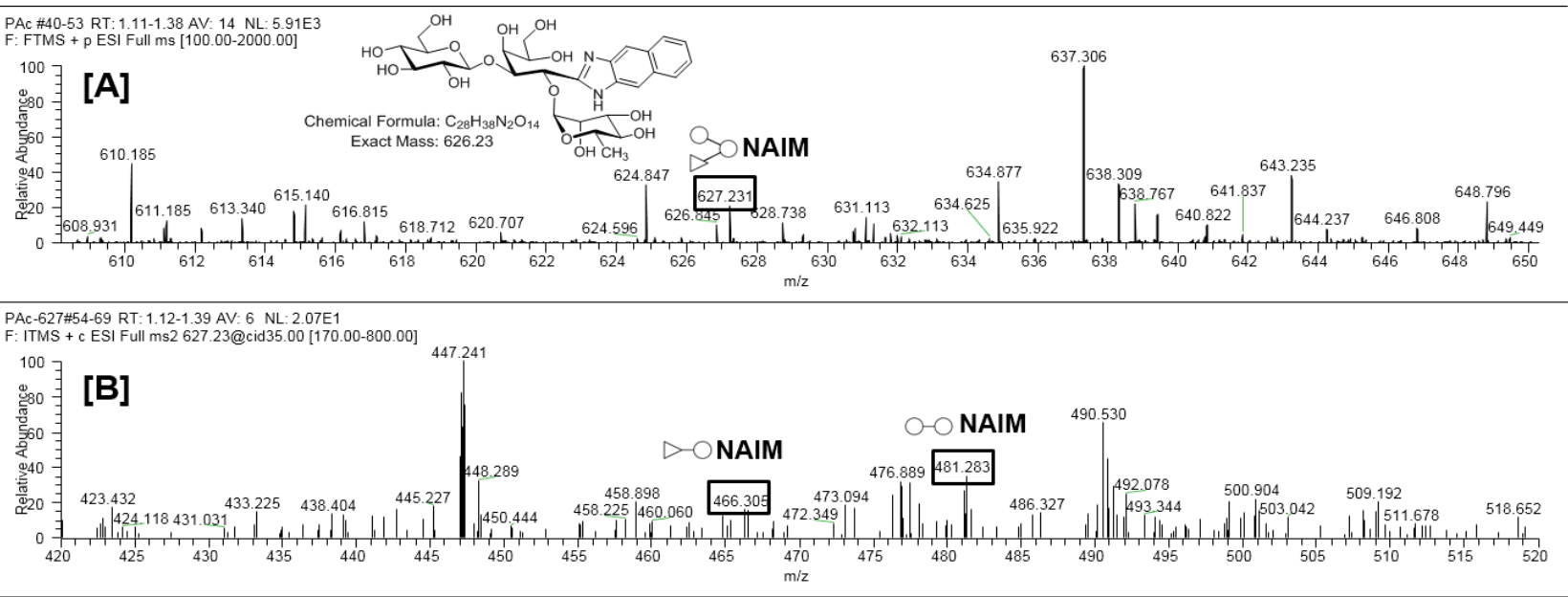

PAc-627\#54-69 RT: $1.12-1.39$ AV: 6 NL: 8.77
F: ITMS + c ESI Full ms2 $627.23 @$ cid 35.00 [170.00-800.00]

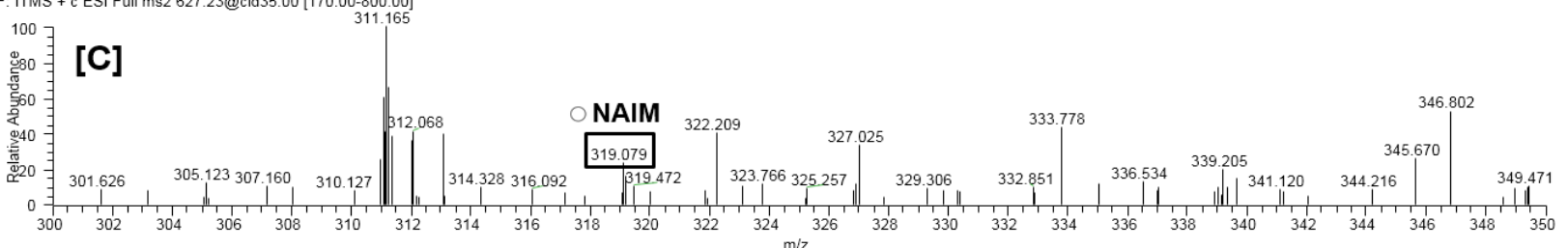

Figure 3. ESI-MS spectrum of the NAIM labeled trisaccharide released from the solanine of the potato Solanum tuberosum (A). ESI-MS/MS spectra demonstrate disaccharide-NAIMs of 481.3 and 465.3 Da (B) and a Hex-NAIM of 319.1 Da (C). 
The glycan released from the solanine constituent in the potato was successfully converted to the NAIM derivative. Figure 3A demonstrates how the NAIM derivative of trisaccharide Glc-GalRha $\left(\mathrm{C}_{28} \mathrm{H}_{38} \mathrm{~N}_{2} \mathrm{O}_{14}, \mathrm{M} . W .626 .6\right)$ corresponds to the $[\mathrm{M}+\mathrm{H}]^{+}$signal at $m / z$ 627.2. To confirm the structure of this glycan, we also carried out the $\mathrm{MS}^{2}$ tandem mass experiment (Figure 3B). For example, the signal at $\mathrm{m} / \mathrm{z} 481.3 \mathrm{might}$ be the result of the elimination of a hexose unit (162 Da), and the signal at $\mathrm{m} / \mathrm{z} 465.3 \mathrm{might}$ be the result of elimination of a deoxyhexose unit (146 Da) from the trisaccharide-NAIM. Removal of one hexose (162 Da) and one deoxyhexose (146 Da) from the disaccharide-NAIM $(\mathrm{m} / \mathrm{z} 481.3$ and $465.3 \mathrm{Da})$ would thereby give a hexose-NAIM ion at $\mathrm{m} / \mathrm{z}$ 319.1 (Figure 3C). This aldo-NAIM, along with the MS method, provides fragment ions of glycan for glycan compositional/structural determination from the released glycan of the plant.

\section{DISCUSSION:}

There are many studies involving the deriving of carbohydrates for analysis using chromatography, electrophoresis and mass spectrometry [16]. The predominant involved focusing on the reducing end labeling of sugars and the used reagents, including 2AB [17], 2AP [18], PMP [19] and etc. In our previous studies, we have demonstrated the use of NAIM tagged sugars in analyses of composition and D-/L-configurations [20]. In the present study, we investigated the composition of common sugars, such as glucose, galactose, lactose, maltose, fructose and sucrose, in beverages and foods. To the best of our knowledge, this is the first report indicating that the aldo-NAIM labeling method is truly useful for determining the sugar composition in drinks and foods.

We further demonstrated that plant glycosides could be treated with orthonaphthalenediamine to form the fluorescent aldo-NAIMs, which not only assisted in LC separation for sugar identification [20], but also enhanced the mass signaling in MALDI-TOF MS measurements [21]. For example, the potato and tomato from Solanaceae family were revealed to contain high percentages of trisaccharide Glc-Gal-Rha and tetrasaccharide HexNAc-HexdeoxyHex-Hex, respectively. Various sugar compositions of natural solanines were also detected by MALDI-TOF MS. In addition to containing alkaloid, varied sugar types and linkages associated with the solanines are also responsible for their biological activities. This study is useful for the structural identification of solanines, especially for the glycan structure. In the current study, only small amounts $(0.1 \mathrm{~g})$ of the raw material were required for the experiment to test the aldo-NAIM labeling method. The aldo-NAIM derivatization enables UV/vis and fluorescence detection, and significantly increases the mass intensity of the target carbohydrate molecules. The conventional method for determining glycosidic linkages via permethylation can also be performed by using the glycan-NAIM derivatives.

Accordingly, the glycan-NAIM derivatives have advantages in investigating the sequence of glycan from various plant glycosides using MS/MS technology. For example, purification of solanines from plants is both difficult and time consuming. We demonstrate in this study that the 
purification of plant extract is no longer needed before fluorescent labeling and mass analysis. Furthermore, we also utilize tandem mass $\left(\mathrm{MS}^{\mathrm{n}}\right)$ and ESI MS technology to confirm the sequence of glycan (Figure 3). It is noted that the solanines isolated from both potato and tomato contain 6deoxy hexose and $N$-acetyl hexosamine. The presence of other glycosides or derivatives in Solanaceae plants, even in small amounts, may lead to interesting biological functions [22, 23]. There are relatively less solanine constituents in the tomato extract compared to that found in the potato, which contains different isomers. However, this difference is not statistically significant when using the same molar amount of samples in mass measurements. The assessment of solanine glycan in the tomato is still ongoing.

\section{CONCLUSIONS}

Our study demonstrates that the sugar composition in food products, including beverages and crop plants (potato and tomato), can be analyzed via the corresponding aldo-NAIM derivatives. Labeling at the reducing end of aldoses is straightforward and efficient, using the iodine-promoted oxidative condensation with 2,3-naphthalenediamine to form the aldo-NAIM derivatives. The fluorescent aldo-NAIM derivatives of saccharides can be analyzed by HPLC on a reversed phase column with improved detection sensitivity. The hydrophobic property of NAIM moiety also enhances the ionization efficiency in MS experiments. This powerful tool is applicable in determining the sugar composition in beverages and dietary foods, in addition to identifying the glycan structures from plant glycosides.

Abbreviations: 2AB, 2-aminobenzamide; 2AP, 2-aminopyridine; ESI-MS/MS, electrospray ionization tandem mass spectrometry; Gal, galactose; Glc, glucose; Hex, hexose; HexNAc, $N$ acetyl hexosamine; HPLC, high performance liquid chromatography; Lacto, lactose; MALDITOF MS, matrix-assisted laser desorption/ionization time of flight mass spectrometry; Malto, maltose; NAIM, naphthylimidazole; PMP, 1-phenyl-3-methyl-5-pyrazolone; Rha, rhamnose; TLC, thin-layer chromatography.

Competing Interests: The authors have no financial interests or conflicts of interests.

Author's Contributions: All authors contributed to this study. Wen-Bin Yang, PhD is the principle investigator for this research and prepared the manuscript. Wei-Ting Hung and Yi-Ting Chen assisted in the food processing and statistical analysis. Shwu-Huey Wang and Yin-Chen Liu are the research coordinators for this study.

Acknowledgments: We thank Professor Jim-Ming Fang at National Taiwan University helped us to revise the manuscript. Mr. Chien-Hung Chen for his technical assistance on MS spectrometry. 
The NAIM kit was provided from Sugarlighter Corporation. This study was supported by Genomics Research Center, Academia Sinica, Taiwan.

\section{REFERECES:}

1. Janket SJ, Buring JE, Manson JE, Liu S, Sesso H. A prospective study of sugar intake and risk of type 2 diabetes in woman. Diabetes Care 2003; 26:1008-1015.

2. Imamura F, O’Connor L, Ye Z, Mursu J, Hayashino Y, Bhupathiraju SN, Forouhi NG. Consumption of sugar sweetened beverages, artificially sweetened beverages, and fruit juice and incidence of type 2 diabetes: systematic review, meta-analysis, and estimation of population attributable fraction. BMJ 2015; 351:h3576.

3. Mckeown G. Epidemiology of colorectal cancer revisited: are serum triglycerides and/or plasma glucose associated with risk? Cancer Epidemiology Biomarkers \& Prevention 1994; 3:687-695.

4. Nishida C, Uavy R, Kumanyika S, Shetty P. The joint WHO/FAO expert consultation on diet, nutrition and the prevention of chronic diseases: process, product and policy implications. Public Health Nutrition 2004; 7:245-250.

5. Scientific report of the 2015 Dietary Guidelines Advisory Committee. Website: http://www.health.gov/dietaryguidelines/2015/.

6. Kell J. Candy maker mars backs labels for added sugar. http://fortune.com/2015/05/08/mars-backslabels-for-added-sugar/.

7. Statement on the Food and Drug Administration's proposed daily value on "Added Sugars". Washington, DC: The Sugar Association; July 24, 2015. http://www.prnewswire.com/news-releases/the-sugar-association-statement-on-the-foodand-drug-administrations-proposed-daily-value-on-added-sugars-300118569.

8. Lin C, Lai PT, Liao KS, Hung WT, Yang WB, Fang JM. Using molecular iodine in direct oxidative condensation of aldoses with diamines: an improved synthesis of aldobenzimidazoles and aldo-naphthimidazoles for carbohydrate analysis. J. Org. Chem. 2008; 73:3848-3853.

9. Hung WT, Wang SH, Chen CH, Fang JM, Yang WB. Tagging $N$-linked glycan with 2,3naphthalenediamine for mass spectrometric analysis. J. Chin. Chem. Soc. 2013; 60:995960.

10. Presenters consider the impact of coming dietary policy changes. IFT15 News. Institute of Food Technologists website. http://news.ift.org/2015/07/13/.

11. Wu F, Tanksley SD. Chromosomal evolution in the plant family Solanaceae. BMC 
Genomics 2010; 11:182.

12. Kim HJ, Baek KH, Lee BW, Choi D, Hur CG. In silico identification and characterization of microRNAs and their putative target genes in Solanaceae plants. Genome 2011; 54:9198.

13. Karas M, Bachmann D, Bahr U, Hillenkamp F. Matrix-assisted ultraviolet laser desorption of non-volatile compounds. Int. J. Mass Spectrom. Ion Processes 1987; 78:53-68.

14. Croxatto A, Prod'hom G, Greub G. Applications of MALDI-TOF mass spectrometry in clinical diagnostic microbiology. FEMS Microbiol. Rev. 2012; 36:380-407.

15. Devkota HP, Joshi KR, Ozasa T, Fukunaga S, Yoshida N, Yahara S. Steroidal glycosides from the fruits, aerial parts and tubers of potato (Solanum tuberosum). J. Pharmacognosy \& Phytochemistry 2015; 3:252-255.

16. Harvey DJ. Derivatization of carbohydrates for analysis by chromatography, electrophoresis and mass spectrometry. J. Chromatogr. B 2011; 879:1196-1225.

17. Bigge, JC, Patel TP, Bruce JA, Goulding PN, Charles SM, Parekh RB. Non-selective and efficient fluorescent labeling of glycans using 2-aminobenzamide and anthranilic acid. Anal. Biochem. 1995; 230:229-238.

18. Fan, JQ; Huynh, LH; Lee, YC. Purification of 2-aminopyridine derivatives of oligosaccharides and related compounds by cation exchange chromatography. Anal. Biochem. 1995; 232:65-68.

19. Saba JA, Shen X, Jamieson JC, Perreault H. Effect of 1-phenyl-3-methyl-5-pyrazolone labeling on the fragmentation behavior of asialo and sialylated $N$-linked glycans under electrospray ionization conditions. Rapid Commun. Mass Spectrom. 1999; 13:704-711.

20. Lin C, Kuo CY, Liao KS, Yang WB. Monosaccharide-NAIM derivatives for D-, Lconfigurational analysis. Molecules 2011; 16:652-664.

21. Lin C, Hung WT, Chen CH, Fang JM, Yang WB. A new naphthimidazole derivative for saccharide labeling with enhanced sensitivity in mass spectrometry detection. Rapid Commun. Mass Spectrom. 2010; 24:85-94.

22. Dafni A, Yaniv Z. Solanaceae as medicinal plants in Israel. J. Ethnopharmacol. 1994; 44:11-18.

23. Ventrella E, Marciniak P, Adamsk Z, Rosinski G, Chowanski S, Falabella P, Scrano L, Bufo SA. Cardioactive properties of Solanaceae plant extracts and pure glycoalkaloids on Zophobas atratus. Insect Science 2015; 22:251-262. 